\title{
Current U.S. splinting practices for individuals with cervical spinal cord injury
}

\author{
Sara Kate Frye ${ }^{1,2} \cdot$ Paula Richley Geigle ${ }^{3}$ \\ Received: 21 November 2019 / Revised: 23 March 2020 / Accepted: 13 May 2020 \\ (c) The Author(s), under exclusive licence to International Spinal Cord Society 2020
}

\begin{abstract}
Study design Survey research design.

Objective To understand current splinting practices of occupational therapists working with individuals with spinal cord injury.

Setting The United States.

Methods An online survey was emailed to occupational therapists working in U.S. inpatient spinal cord rehabilitation facilities. The survey included questions about hand splinting practices in their patients with cervical spinal cord injury.

Results Sixty-five occupational therapists in 21 different states completed the survey. They reported that current and predicted hand function was the primary principle guiding splint decision making. Across all levels of cervical SCI, resting hand splints are commonly prescribed for night use, and $64.6 \%$ of respondents stated they typically recommend them for individuals without active arm movement. Most respondents (73.8\%) also report prescribing wrist splints for day use for individuals without active wrist movement. Survey results indicate that therapists are using splints less frequently overall for all levels of injury. The longopponens splint is no longer being used regularly in SCI and the MCP block splint is being used more frequently.

Conclusion Survey responses indicated that splinting is standard care for individuals with cervical spinal cord injury and that the level of SCI dictates specific recommendations. Splint practice guidelines are a framework for intervention mediated by case-specific clinical reasoning and client input.
\end{abstract}

\section{Introduction}

More than 17,000 Americans incur a spinal cord injury (SCI) each year, and almost $60 \%$ of these injuries affect the cervical spinal cord [1-3]. Regardless of the severity of the injury, individuals with cervical SCI typically experience motor and sensory impairments in all four limbs and require intensive acute rehabilitation to promote independence in activities of daily living (ADL) [2]. People with cervical SCI report recovery of hand function is of primary importance among the profound long-term effects of SCI [3, 4].

Sara Kate Frye

sfryeOT@gmail.com

1 University of Maryland Rehabilitation \& Orthopaedic Institute, Baltimore, MD, USA

2 Thomas Jefferson University Hospital, Philadelphia, PA, USA

3 Department of Physical Therapy, South College, Knoxville, TN, USA
The International Spinal Cord Injury Upper Extremity Basic Data Set states that splints are essential for individuals with cervical SCI to achieve maximal hand function [5]. Splinting prepares the hand for functional use via compensatory strategies, such as tenodesis, in the event of natural recovery, surgical intervention via nerve or tendon transfers, and for future novel therapy advances [6]. Aggressive upper extremity management is required to promote hand function, especially for the $47.6 \%$ of individuals with SCI who have incomplete cervical SCI and in whom the potential for neurologic recovery is greater [1].

However, the evidence for hand splinting is limited. The search engine of a university library system was used to locate full-text articles using the following terms to inform this research project: splint, orthosis, orthotic, SCI, tetraplegia, quadriplegia, and tenodesis. Philosophies and constructs of long-term splint use for individuals living with cervical SCI are not well-documented, with the most recent splinting practice data published in the 1990s [7-9]. Furthermore, the primary studies cited in the 1990s articles were published in the 1960s and 1970s. Data collected in 1991 identified four 
primary functions of static hand splints for individuals with cervical SCI [7]. These identified primary factors include (a) prevention of overstretching, particularly wrist extensors; (b) maintenance of a functional position; (c) prevention of deformities such as claw hand; and (d) protection and stabilization of flail joints. A 1991 interventional study found no significant difference in hand function in a group of 13 individuals with C6 cervical SCI who used a static splint at night versus no orthosis use [8]. Additional splint data collected in 1994 from 46 international SCI rehabilitation centers indicates, resting hand splints were prescribed to promote functional positioning, maintain joint integrity, support arches, prevent muscle overstretching, and maintain webspace [9]. Prescribed wrist splints typically promoted functional positioning and prevented muscle overstretching.

Recommended current standard care after cervical SCI includes hand splinting as soon as possible after injury and continuing throughout rehabilitation; however, limited standard protocols exist across the continuum of care. One protocol proposed that optimal muscle shortening for tenodesis is achieved by splints issued immediately after injury and worn for 23 hours daily until the onset of rehabilitation with breaks only for range of motion exercises [10]. Another suggested a protocol of (1) resting hand splints for night time/rest when wrist and digit strength is $0-3 / 5$; (2) futuro wrist splints for daytime hand use for individuals with elbow flexion against gravity but weak wrists and hands; and (3) hand based or thumb splints and/ or taping of the digits at night/rest for individuals with wrist extension against gravity but no digit movement [10].

Because contemporary cervical SCI splint use data are lacking, we initiated a survey of current real-life splinting practices.

\section{Methods}

\section{Participants}

We distributed our survey to occupational therapists specializing in SCI and working in Commission on
Accreditation of Rehabilitation Facilities (CARF)-accredited SCI programs in the United States, and to occupational therapists who are members of the American Spinal Injury Association (ASIA).

\section{Instrument}

We initiated survey development with a literature review of previous splinting surveys [7, 9]. Splint practice guidelines and clinical indications described in previous studies informed the development of our survey. Then we developed an electronic survey to examine clinical use of the five common spinal cord rehabilitation splints: resting hand, wrist, long opponens, short opponens, and metacarpalphalangeal (MCP) blocking splints. This online survey was piloted successfully by an occupational therapist who reported no difficulty interpreting and responding, and no obvious survey omissions.

The survey consisted of two parts: a demographics section and a clinical section. The demographic section consisted of four questions, and the clinical section consisted of questions on current splinting practices. Specifically, the clinical section of the survey reviewed the positional and functional splinting practices for each level of injury $\mathrm{C} 1-\mathrm{C} 4, \mathrm{C} 5, \mathrm{C} 6$, and $\mathrm{C} 7-8$, identifying the main factors dictating splint usage, rating criteria included in splint decision making, and logistics surrounding splint prescription and fabrication. Questions consisted of multiple choice and Likert scale items with options for an open response if answer options did not describe their current practice. See Fig. 1 for sample survey questions with one of each type of clinical question demonstrated.

\section{Survey distribution}

To obtain e-mail addresses for dissemination, we attempted to reach clinicians from all centers identified on the CARF website by telephone. An electronic survey was emailed to the occupational therapy department at all SCI rehabilitation centers where we established telephone contact. In addition,
Fig. 1 Sample survey items from the electronic survey sent to occupational therapists.

\section{Sample Survey Items}

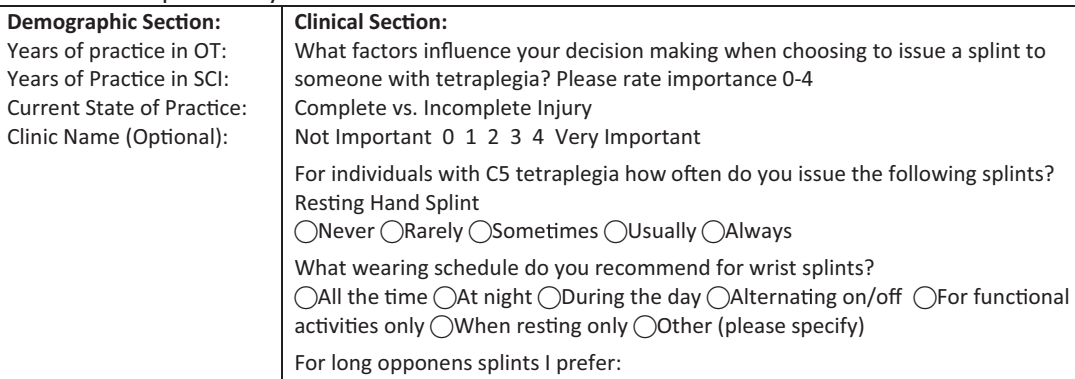


we emailed the survey to all occupational therapists who held membership in ASIA. Survey responses were collected over a 1-year period. Participants were not compensated for survey completion.

\section{Data analysis}

An online survey platform gathered the data into a spreadsheet format for basic statistical analysis. Surveys were considered incomplete and were not included in the final analysis if the demographics portion was completed, but the clinical portion was not. After distribution, 97 occupational therapists participated in the survey, 65 of whom completed the clinical section of the survey for data analysis inclusion. Participants did not need to answer all questions of the survey for inclusion in the analysis, and some section information was omitted based on previous answers. For example, questions about wrist splints were omitted if the participant reported they did not use them in practice.

\section{Results}

\section{Participants}

Sixty-five occupational therapists practicing in 21 states completed the survey: 28 practiced in the Northeast, 12 in the Midwest, 13 in the South, and 11 in the West. Their general practice experience and SCI-specific experience ranged from $<1$ year to 30 years (mean $=12.07$ years of OT experience and 9.52 years practicing in $\mathrm{SCI}$ ).

\section{Splinting determinants}

Hand function was the primary factor that influenced therapist decision making when determining whether an individual with SCI would benefit from splinting, with $80 \%$ of therapists rating it as very important (Table 1). Additional factors impacting their decision making comprised preventing contractures and maintaining an optimal range of motion, especially when spasticity was present. Other key factors guiding decision making incorporated preventing overstretching of wrist extensors and maintaining optimal position for functional grasp or tenodesis. Notably, reimbursement influenced therapist decision making the least, with 32 of 64 respondents stating it did not affect their decision making at all.

Table 2 shows splint use frequency, in particular, how many therapists usually or always use a splint based on the level of injury. Table 3 shows another view of this data using a weighted average. Resting hand splints were used more frequently for higher levels of injury; wrist splints and long opponens for injuries at the C5 level; short opponens for injuries at the C6 level; and MP blocking splints for injuries at the $\mathrm{C} 7$ level.

\section{Resting hand splints}

The most in-depth survey questions examined resting hand splint usage. Custom-made splints are preferred by 28 of 52 respondents who regularly provided resting hand splints. The typical wearing schedule for resting hand splints included night time use, with wearing tolerance increased over a few days. Some therapists reported utilizing an alternating wear schedule for individuals who demonstrated poor splint tolerance. Clinical fabrication criteria included palmar arch preservation and hand preparation for functional activities by maintaining thumb opposition. Clinicians reported customizing splint position based on tone and whether the future expectation was a nonfunctional hand, a tenodesis grasp, or regaining active finger motion.

Participants indicate employing slight position variations for resting hand splints. However, most respondents report positioning the wrist in $10-40^{\circ}$ extension $\left(30^{\circ}\right.$ most frequent). Digits are positioned in some flexion: $\mathrm{MCP}$ at $0-90$ (70-90 most typical); proximal interphalangeal (PIP) 0-75 (10-30 most typical); distal interphalangeal (DIP) 0-70 (10-20 most typical). The thumb is typically positioned in opposition. Notably, one therapist reports an alternate splinting position preparing the hand for the release phase of tenodesis: $25^{\circ}$ wrist flexion, $25^{\circ} \mathrm{MCP}$ flexion, and $10^{\circ}$ PIP/DIP flexion.

Of the respondents reporting fabrication of hand splint, most ( 38 of 43) indicate hand splint construction takes $<1 \mathrm{~h}$, and 11 of these therapists require less than $30 \mathrm{~min}$ for fabrication time. Patient splint education typically occurs in under $30 \mathrm{~min}$ (37 of 44 respondents) and never exceeds $1 \mathrm{~h}$. Sixty-five percent of respondents report splinting costs are imbedded in the hospital stay cost while 35\% report issuing an additional, separate splint charge.

\section{Wrist splints}

Prefabricated wrist splints are preferred by 29 of 48 clinicians who regularly prescribe wrist splints to be worn during the day to increase functional activity participation. Dorsal varieties and a U-Cuff are mentioned as favorite options. The primary wrist splint goal is to prevent overstretching of the wrist extensors, and the addition of a universal cuff provides a stable base for ADLs.

\section{Long opponens}

Long-opponens splint use occurs less frequently than other splints, with custom fabricated splints preferred by 16 of 18 
Table 1 Factors influencing splint decision making.

\begin{tabular}{|c|c|c|c|c|c|c|c|}
\hline Factor influencing splinting & 0 Not important & 1 & 2 & 3 & 4 Very important & $N$ & Weighted average \\
\hline Hand function & $\begin{array}{l}0.00 \% \\
0\end{array}$ & $\begin{array}{l}0.00 \% \\
0\end{array}$ & $\begin{array}{l}1.54 \% \\
1\end{array}$ & $\begin{array}{l}18.46 \% \\
12\end{array}$ & $\begin{array}{l}80.00 \% \\
52\end{array}$ & 65 & 4.78 \\
\hline Prevent contractures of wrist and hand & $\begin{array}{l}0.00 \% \\
0\end{array}$ & $\begin{array}{l}0.00 \% \\
0\end{array}$ & $\begin{array}{l}7.81 \% \\
5\end{array}$ & $\begin{array}{l}26.56 \% \\
17\end{array}$ & $\begin{array}{l}65.63 \% \\
42\end{array}$ & 64 & 4.58 \\
\hline Spasticity & $\begin{array}{l}1.54 \% \\
1\end{array}$ & $\begin{array}{l}0.00 \% \\
0\end{array}$ & $\begin{array}{l}9.23 \% \\
6\end{array}$ & $\begin{array}{l}26.15 \% \\
17\end{array}$ & $\begin{array}{l}63.08 \% \\
41\end{array}$ & 65 & 4.49 \\
\hline Protect and stabilize joints & $\begin{array}{l}1.54 \% \\
1\end{array}$ & $\begin{array}{l}0.00 \% \\
0\end{array}$ & $\begin{array}{l}7.69 \% \\
5\end{array}$ & $\begin{array}{l}30.77 \% \\
20\end{array}$ & $\begin{array}{l}60.00 \% \\
39\end{array}$ & 65 & 4.48 \\
\hline $\begin{array}{l}\text { Prevent overstretching of wrist } \\
\text { extensors }\end{array}$ & $\begin{array}{l}0.00 \% \\
0\end{array}$ & $\begin{array}{l}3.08 \% \\
2\end{array}$ & $\begin{array}{l}10.77 \% \\
7\end{array}$ & $\begin{array}{l}30.77 \% \\
20\end{array}$ & $\begin{array}{l}55.38 \% \\
36\end{array}$ & 65 & 4.38 \\
\hline Range of motion & $\begin{array}{l}0.00 \% \\
0\end{array}$ & $\begin{array}{l}1.54 \% \\
1\end{array}$ & $\begin{array}{l}9.23 \% \\
6\end{array}$ & $\begin{array}{l}38.46 \% \\
25\end{array}$ & $\begin{array}{l}50.77 \% \\
33\end{array}$ & 65 & 4.38 \\
\hline Patient acceptance/follow through & $\begin{array}{l}0.00 \% \\
0\end{array}$ & $\begin{array}{l}3.08 \% \\
2\end{array}$ & $\begin{array}{l}16.92 \% \\
11\end{array}$ & $\begin{array}{l}40.00 \% \\
26\end{array}$ & $\begin{array}{l}40.00 \% \\
26\end{array}$ & 65 & 4.17 \\
\hline Maintain passive range & $\begin{array}{l}1.54 \% \\
1\end{array}$ & $\begin{array}{l}4.62 \% \\
3\end{array}$ & $\begin{array}{l}15.38 \% \\
10\end{array}$ & $\begin{array}{l}40.00 \% \\
26\end{array}$ & $\begin{array}{l}38.46 \% \\
25\end{array}$ & 65 & 4.09 \\
\hline Maintain thumb webspace & $\begin{array}{l}0.00 \% \\
0\end{array}$ & $\begin{array}{l}6.25 \% \\
4\end{array}$ & $\begin{array}{l}18.75 \% \\
12\end{array}$ & $\begin{array}{l}37.50 \% \\
24\end{array}$ & $\begin{array}{l}37.50 \% \\
24\end{array}$ & 64 & 4.06 \\
\hline Level of injury & $\begin{array}{l}0.00 \% \\
0\end{array}$ & $\begin{array}{l}10.94 \% \\
7\end{array}$ & $\begin{array}{l}9.38 \% \\
6\end{array}$ & $\begin{array}{l}45.31 \% \\
29\end{array}$ & $\begin{array}{l}34.38 \% \\
22\end{array}$ & 64 & 4.03 \\
\hline Promote optimal tightening of the hand & $\begin{array}{l}6.15 \% \\
4\end{array}$ & $\begin{array}{l}7.69 \% \\
5\end{array}$ & $\begin{array}{l}12.31 \% \\
8\end{array}$ & $\begin{array}{l}27.69 \% \\
18\end{array}$ & $\begin{array}{l}46.15 \% \\
30\end{array}$ & 65 & 4.00 \\
\hline Maintain arches of hand & $\begin{array}{l}0.00 \% \\
0\end{array}$ & $\begin{array}{l}7.69 \% \\
5\end{array}$ & $\begin{array}{l}29.23 \% \\
19\end{array}$ & $\begin{array}{l}32.31 \% \\
21\end{array}$ & $\begin{array}{l}30.77 \% \\
20\end{array}$ & 65 & 3.86 \\
\hline Correct deformity & $\begin{array}{l}0.00 \% \\
0\end{array}$ & $\begin{array}{l}12.31 \% \\
8\end{array}$ & $\begin{array}{l}18.46 \% \\
12\end{array}$ & $\begin{array}{l}41.54 \% \\
27\end{array}$ & $\begin{array}{l}27.69 \% \\
18\end{array}$ & 65 & 3.85 \\
\hline Family acceptance/follow through & $\begin{array}{l}1.54 \% \\
1\end{array}$ & $\begin{array}{l}7.69 \% \\
5\end{array}$ & $\begin{array}{l}27.69 \% \\
18\end{array}$ & $\begin{array}{l}40.00 \% \\
26\end{array}$ & $\begin{array}{l}23.08 \% \\
15\end{array}$ & 65 & 3.75 \\
\hline Medical complications & $\begin{array}{l}1.54 \% \\
1\end{array}$ & $\begin{array}{l}9.23 \% \\
6\end{array}$ & $\begin{array}{l}30.77 \% \\
20\end{array}$ & $\begin{array}{l}40.00 \% \\
26\end{array}$ & $\begin{array}{l}18.46 \% \\
12\end{array}$ & 65 & 3.65 \\
\hline Complete vs. incomplete injury & $\begin{array}{l}1.56 \% \\
1\end{array}$ & $\begin{array}{l}18.75 \% \\
12\end{array}$ & $\begin{array}{l}25.00 \% \\
16\end{array}$ & $\begin{array}{l}28.13 \% \\
18\end{array}$ & $\begin{array}{l}26.56 \% \\
17\end{array}$ & 64 & 3.59 \\
\hline Preserve the hand for future surgery & $\begin{array}{l}9.23 \% \\
6\end{array}$ & $\begin{array}{l}10.77 \% \\
7\end{array}$ & $\begin{array}{l}21.54 \% \\
14\end{array}$ & $\begin{array}{l}29.23 \% \\
19\end{array}$ & $\begin{array}{l}29.23 \% \\
19\end{array}$ & 65 & 3.58 \\
\hline Time since injury & $\begin{array}{l}6.15 \% \\
4\end{array}$ & $\begin{array}{l}18.46 \% \\
12\end{array}$ & $\begin{array}{l}32.31 \% \\
21\end{array}$ & $\begin{array}{l}33.85 \% \\
22\end{array}$ & $\begin{array}{l}9.23 \% \\
6\end{array}$ & 65 & 3.22 \\
\hline Concerns about nursing follow through & $\begin{array}{l}15.87 \% \\
10\end{array}$ & $\begin{array}{l}17.46 \% \\
11\end{array}$ & $\begin{array}{l}22.22 \% \\
14\end{array}$ & $\begin{array}{l}30.16 \% \\
19\end{array}$ & $\begin{array}{l}14.29 \% \\
9\end{array}$ & 63 & 3.1 \\
\hline Improve appearance of hand & $\begin{array}{l}20.31 \% \\
13\end{array}$ & $\begin{array}{l}31.25 \% \\
20\end{array}$ & $\begin{array}{l}26.56 \% \\
17\end{array}$ & $\begin{array}{l}17.19 \% \\
11\end{array}$ & $\begin{array}{l}4.69 \% \\
3\end{array}$ & 64 & 2.55 \\
\hline Reimbursement & $\begin{array}{l}50.00 \% \\
32\end{array}$ & $\begin{array}{l}26.56 \% \\
17\end{array}$ & $\begin{array}{l}15.63 \% \\
10\end{array}$ & $\begin{array}{l}4.69 \% \\
3\end{array}$ & $\begin{array}{l}3.13 \% \\
2\end{array}$ & 64 & 1.84 \\
\hline
\end{tabular}

clinicians who report regular long opponens use. Clinicians state these splints are recommended for functional activities but also note potential interference with power wheelchair operation.

\section{Short opponens}

Therapists who report regular short opponens use prefer to fabricate custom splints almost exclusively (41 of 43 respondents). These splints facilitate tenodesis by opposing the thumb and preventing thumb overstretching during functional tasks.

\section{MP blocking splint}

All 24 clinicians who report regular MP blocking splint use fabricate custom splints to prevent MP hyperextension during functional hand tasks. 


\section{Other splints}

In addition to the five common splints listed in the survey, therapists describe commonly using the following additional devices: elbow extension splints, elbow pillow splints, anti-spasticity splints, palm splints, intrinsic plus or minus splints, tone and positioning splints, tenodesis splints or orthotics, and finger flexion gloves or mitts.

\section{Discussion}

A literature review reveals limited and dated evidence on splinting practices for individuals with SCI. Data on current

Table 2 Number of therapists who report they usually/always provide a given splint by level of injury $(N=65)$.

\begin{tabular}{lllll}
\hline & $\mathrm{C} 1-4$ & $\mathrm{C} 5$ & $\mathrm{C} 6$ & $\mathrm{C} 7-8$ \\
\hline Resting hand splint & 42 & 38 & 22 & 8 \\
& $20 / 22$ & $23 / 15$ & $15 / 7$ & $5 / 3$ \\
Wrist splint & 26 & 48 & 29 & 9 \\
& $18 / 8$ & $33 / 15$ & $22 / 7$ & $7 / 2$ \\
Long-opponens splint & 4 & 9 & 4 & 1 \\
& $3 / 1$ & $6 / 3$ & $2 / 2$ & $1 / 0$ \\
Short-opponens splint & 0 & 8 & 19 & 16 \\
& $0 / 0$ & $7 / 1$ & $13 / 6$ & $14 / 2$ \\
MCP blocking splint & 1 & 2 & 3 & 11 \\
& $1 / 0$ & $2 / 0$ & $13 / 0$ & $10 / 1$ \\
\hline
\end{tabular}

Table 3 Weighted average of splint use by level of injury.

\begin{tabular}{lllll}
\hline & C1-4 & C5 & C6 & C7-8 \\
\hline Resting hand splint & 3.77 & 3.57 & 2.27 & 2.58 \\
Wrist splint & 3.28 & 3.88 & 3.25 & 2.52 \\
Long-opponens splint & 1.94 & 2.36 & 2.22 & 1.81 \\
Short-opponens splint & 1.75 & 2.38 & 3.06 & 2.86 \\
MCP blocking splint & 1.74 & 1.97 & 2.06 & 2.37 \\
Other & 2.50 & 2.53 & 2.58 & 2.47 \\
\hline
\end{tabular}

$0=$ never, $1=$ rarely, $2=$ sometimes, $3=$ usually, $4=$ always. splinting trends in U.S. SCI rehabilitation centers offer care standardization guidance for therapists who provide services to individuals with SCI at nonspecialized centers across the continuum of care. Furthermore, our data on current practice usage facilitates upper extremity orthotic protocol development to ensure optimal outcomes for individuals with cervical SCI.

Our survey study updates knowledge on the splinting practices of occupational therapists working in specialized SCI programs in the United States. Our findings align with many of the prior published reports on hand splinting practices in individuals with SCI [7-9]. In particular, the resting hand splint functional position in our results concurred with literature published 30 and 40 years ago with ideal wrist position at $0-45^{\circ}$ extension; MCP at $90^{\circ}$ of flexion; and DIP/ PIP at $0-30^{\circ}$ of flexion [7]. Our results also provide insight into how splinting practices for SCI have changed in recent decades. See Table 4 for a comparison of current data to previous survey data collected in 1992 [7].

Overall, occupational therapists are using splints less frequently for all levels of SCI. One significant change is resting hand splint usage decreased for all levels of injuries. The long-opponens splint is being used infrequently because when compared with a standard wrist splint, the long opponens may impede bimanual grasp and universal cuff use. One exception is the MCP block splint, which is recommended more frequently in our research for all levels of injury.

Splint use reduction may be attributed to changes in the healthcare delivery model. Decreasing current inpatient rehabilitation length of stay (LOS) may influence clinicians to prioritize more restorative interventions, such as functional electrical stimulation over splinting, particularly for those individuals with incomplete injuries [11]. This shorter inpatient (LOS) does not allow for an initial period of conservative wrist extension strengthening while using a rest period wrist splint for individuals who are developing a tenodesis grasp.

Our findings indicate splinting guidelines should not be rigidly prescriptive, but instead stem from a conceptual, clinical, and reasoning framework. Therapists with less clinical experience may seek out and follow established splint protocols, while more experienced therapists are more likely
Table 4 Percentage of therapists recommending splint use by level of injury: Splinting data comparison between Krajnik \& Bridle's work in 1992 [7] to our data gathered in 2018.

\begin{tabular}{|c|c|c|c|c|c|c|c|}
\hline & & $\begin{array}{l}\text { Resting } \\
\text { hand }\end{array}$ & $\begin{array}{l}\text { Long } \\
\text { opponens }\end{array}$ & $\begin{array}{l}\text { Short } \\
\text { opponens }\end{array}$ & $\begin{array}{l}\text { Dorsal } \\
\text { wrist }\end{array}$ & $\begin{array}{l}\text { Wrist } \\
\text { cock-up }\end{array}$ & $\begin{array}{l}\text { MCP } \\
\text { block }\end{array}$ \\
\hline \multirow[t]{2}{*}{ C5 } & 1992 & 87.1 & 47.5 & 4 & 49.5 & 49.5 & 0 \\
\hline & 2018 & 58.5 & 13.8 & 12.3 & \multicolumn{2}{|c|}{73.8} & 3 \\
\hline \multirow[t]{2}{*}{ C6 } & 1992 & 67.3 & 25.7 & 46.5 & 33.7 & 40.6 & 3 \\
\hline & 2018 & 33.8 & 6.2 & 29.2 & \multicolumn{2}{|c|}{44.6} & 4.6 \\
\hline \multirow[t]{3}{*}{ C7-8 } & C7 1992 & 41.6 & 8.9 & 55.4 & 13.9 & 19.8 & 5.9 \\
\hline & C8 1992 & 17.8 & 3 & 37.6 & 4 & 10.9 & 6.9 \\
\hline & C7-8 2018 & 12.3 & 1.5 & 24.6 & \multicolumn{2}{|c|}{13.8} & 16.9 \\
\hline
\end{tabular}


Fig. 2 A model for splint decision making.

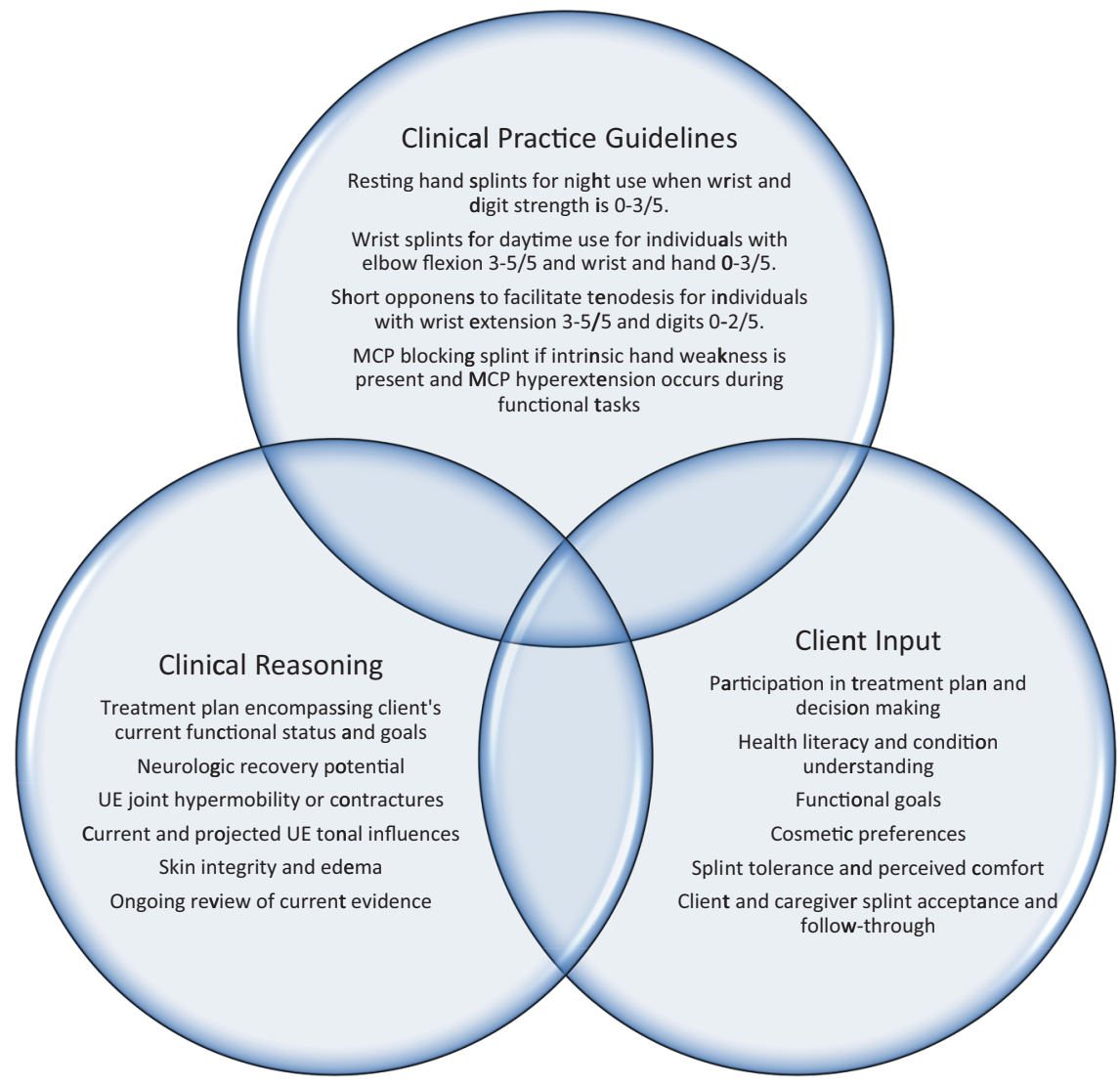

to create individualized splinting programs [12]. Clinical decision making references practice guidelines, but it is critical to realize each client is unique. Clinicians should seek input from the client while using case-specific clinical reasoning to ensure the splinting process is effective. Patient goals, neurological function, strength, and functional abilities may change overtime so splint recommendations should be modified as client skills and needs evolve. Figure 2 illustrates the interplay of clinical practice guidelines, clinical reasoning, and client input for splint decision making.

\section{Limitations}

The survey outcome is potentially limited by clinician time constraints as well as the inability to query specific responses for increased understanding. Ideally, additional information about splint use would improve the outcome depth and scope; however, the survey completion time was limited to 20 minutes or less to acquire more responses and not overtax practitioners. Another limitation of this survey is the inherent difficulty of distributing the survey to occupational therapy practitioners at SCI centers. The methodology of establishing phone contact was time challenging as department phone numbers are typically not listed on websites. We found hospital operators may not know therapy department extensions, and clinicians have limited time to answer and return phone calls. Based on the geographic distribution of the sample, our results may not reflect all SCI rehabilitation splinting practice; however, they do provide an important snapshot of current practice.

\section{Future research directions}

To increase knowledge depth, interviews and focus group research could generate more detailed data on the prevalence and clinical decision making related to dynamic splint use and neuro-prosthesis prescription for SCI. Future studies could contrast acute rehabilitation recommendations with long-term use of orthoses across the lifespan and continuum of care. Questions also remain about financial costs for fabrication and re-fabrication and differences between custom and off-the-shelf splints. As technology continues to advance, the role $3 \mathrm{D}$ printing could play a role in splinting and may offer additional opportunities for lowcost custom and potentially specialized orthotics [13].

\section{Conclusion}

This survey indicated splinting is considered standard care for individuals with cervical SCI. In particular, resting hand splints are commonly recommended for night use for all 
levels of cervical SCI, and wrist splints are commonly recommended for day use for those without active wrist movement. In addition, short-opponens splints can be used to facilitate tenodesis in individuals with wrist extension and MCP blocking splints can be prescribed to promote emerging grasp patterns. Splint practice guidelines should be used as a framework for intervention guided by clinical reasoning and client input.

Acknowledgements Thank you, Peter Gorman, MD, for your support.

Funding This work is supported by the Craig H. Neilsen Foundation Allied Health Professional Research Award of the American Spinal Injury Association (ASIA).

\section{Compliance with ethical standards}

Conflict of interest The authors declare that they have no conflict of interest.

Ethical approval We certify that all applicable institutional and governmental regulations concerning the ethical use of human volunteers were followed during this research. This research was determined not to be human subject research by the University of Maryland, Baltimore IRB Submission HP-00074550 and received an exemption during Institutional Review Board (IRB) review.

Publisher's note Springer Nature remains neutral with regard to jurisdictional claims in published maps and institutional affiliations.

\section{References}

1. National Spinal Cord Injury Statistical Center (NSCISC). Spinal cord injury facts and figures at a glance. 2018. https://www.nscisc.
uab.edu/Public/Facts\%20and\%20Figures\%202019\%20-\%20Fina 1.pdf.

2. Hartnett A, Rice D, McIntyre A, Mehta S, Iruthayarajah I, Benton I, et al. In: Eng JJ, editor. Spinal Cord Injury Rehabilitation Evidence Version 70. 2019. p. 1-137.

3. Anderson KD. Targeting recovery: priorities of the spinal cord injured population. J Neurotrauma. 2004;21:1371-83.

4. Snoek GJ, IJzerman MJ, Post MW, Stiggelbout AM, Roach MJ, Zilvold G. Choice-based evaluation for the improvement of upperextremity function compared with other impairments in tetraplegia. Arch Phys Med Rehabil. 2005;86:1623-30.

5. Biering-Sørensen F, Bryden A, Curt A, Friden J, Harvey LA, Mulcahey MJ, et al. International spinal cord injury upper extremity basic data set. Spinal Cord. 2014;52:652-7.

6. Harvey LA, Lin C-W, Glinsky JV, De Wolf A. The effectiveness of physical interventions for people with spinal cord injuries: a systematic review. Spinal Cord. 2009;47:184-95.

7. Krajnik SR, Bridle MJ. Hand splinting in quadriplegia: current practice. Am J Occup Ther. 1992;46:149-56.

8. DiPasquale-Lehnerz P. Orthotic intervention for development of hand function with C-6 quadriplegia. Am J Occup Ther. 1994;48:138-44.

9. Curtin M. Development of a tetraplegic hand assessment and splinting protocol. Paraplegia. 1994;32:159-69.

10. Harvey L. Principles of conservative management for a nonorthotic tenodesis grip in tetraplegics. J Hand Ther. 1996;9: 238-42.

11. Tschoepe R, Benfield A, Mercer V, Posey R. Systematic review of community transition programs after acute rehabilitation for adults with traumatic spinal cord injury. Am J Occup Ther. 2019;73 (4_Supplement_1):7311510245p1.

12. Chazen L-A, Franzsen D. Expert opinion on splinting adult patients with neurological injuries. South Afr J Occup Ther. 2016;46:4-9. https://doi.org/10.17159/2310-3833/2016/v46n2a2.

13. Portnova AA, Mukherjee G, Peters KM, Yamane A, Steele KM. Design of a 3D-printed, open-source wrist-driven orthosis for individuals with spinal cord injury. PLOS ONE. 2018;13:1-18. https://www.ncbi.nlm.nih.gov/pmc/articles/ PMC5823450/. 